\title{
Comparison between Fovea-Sparing and Complete Internal Limiting Membrane Peeling for the Treatment of Myopic Traction Maculopathy: A Systemic Review and Meta-Analysis
}

\author{
Qiaozhu Zeng ${ }^{a, b}$ Yuou Yao ${ }^{a, b}$ Mingwei Zhao ${ }^{a, b}$ \\ aDepartment of Ophthalmology, Eye Diseases and Optometry Institute, Peking University People's Hospital, Beijing, \\ China; 'beijing Key Laboratory of Diagnosis and Therapy of Retinal and Choroid Diseases, College of Optometry, \\ Peking University Health Science Center, Beijing, China
}

\section{Keywords}

Internal limiting membrane peeling $\cdot$ Vitrectomy $\cdot$ Meta ·

Myopic traction maculopathy

\begin{abstract}
Introduction: Myopic traction maculopathy (MTM) is a major cause of impaired vision in eyes with high myopia, which is characterized by retinal thickening, retinoschisis, lamellar macular hole $(\mathrm{MH})$, and foveal retinal detachment. Pars plana vitrectomy (PPV) with fovea-sparing internal limiting membrane peeling (ILMP) has been developed to theoretically prevent postoperative $\mathrm{MH}$ formation and improve best-corrected visual acuity (BCVA) gain for MTM compared with the complete ILMP. However, in previous studies, the anatomic and visual outcomes still remain uncertain and controversial. Objectives: The aim of this study was to evaluate the anatomic and visual outcomes of vitrectomy with fovea-sparing ILMP for the treatment of MTM compared with complete ILMP. Methods: Articles from PubMed, EMBASE, Web of Science, and Cochrane Library were systematically retrieved. The main outcomes were the rate of a postoperative $\mathrm{MH}$ and visual improvement of BCVA (converted to logarithm of the minimum angle of resolution [logMAR]). The secondary outcomes were the proportion of patients with visual improvement, the proportion of anatomic success,
\end{abstract}

preoperative and postoperative BCVA, preoperative and postoperative central fovea thickness, and time to anatomic resolution. Results: There was a higher rate of postoperative $\mathrm{MH}$ formation (odds ratio [OR] 5.64; 95\% confidence interval [Cl]: 1.72-18.44; $p=0.004$ ) and less improvement of BCVA in logMAR (mean difference [MD] $-0.09 ; 95 \% \mathrm{Cl}$ : -0.18 to 0.00 ; $p=0.04$ ) in the complete ILMP group. However, postoperative BCVA (MD 0.14; 95\% Cl: 0.00-0.27; $p=0.05$ ), the proportion of patients with visual improvement (OR 0.39; $95 \% \mathrm{Cl}$ : $0.15-1.02 ; p=0.05)$, postoperative central foveal thickness (MD $-10.02 ; 95 \% \mathrm{Cl}:-24.4$ to $4.36 ; p=0.17)$, the rate of anatomic success (MD 0.39; 95\% Cl:0.15-1.03; $p=0.06$ ), and time to resolution (MD $-1.65 ; 95 \% \mathrm{Cl}$ : -3.66 to $0.36 ; p=0.11$ ) showed no significant differences. Conclusion: PPV combined with the fovea-sparing ILMP could contribute to a lower MH formation rate and more improvement of BCVA in logMAR than PPV combined with complete ILMP.

(C) 2021 The Author(s).

Published by S. Karger AG, Basel

\section{Introduction}

Myopic traction maculopathy (MTM) is a major cause of impaired vision in eyes with high myopia, which is characterized by retinal thickening, retinoschisis, lamellar macular hole $(\mathrm{MH})$, and foveal retinal detachment [1-
(C) 2021 The Author(s)

Published by S. Karger AG, Basel

This is an Open Access article licensed under the Creative Commons Attribution-NonCommercial-4.0 International License (CC BY-NC) (http://www.karger.com/Services/OpenAccessLicense), applicable to the online version of the article only. Usage and distribution for commercial purposes requires written permission.
Correspondence to:

Mingwei Zhao,drzmwpku@163.com 
3]. The prevalence of MTM is $9-34.4 \%$ in highly myopic eyes [2-4], with a spontaneous resolution rate of only $0-3.9 \%$ [5-7]. During general natural course, MTM starts with the retinoschisis thickening, followed by foveal detachment, then progression to a full-thickness $\mathrm{MH}$ and $\mathrm{MH}$ with retinal detachment [5-7] as a consequence of collective retinal traction from the adherent vitreous cortex, epiretinal membrane, internal limiting membrane (ILM), retinal vessels, and posterior staphyloma [5-10].

Therefore, surgery to release retinal traction is theoretically of pivotal importance in resolving MTM and preventing $\mathrm{MH}$ and devastating vision loss. The widely performed surgery for MTM is pars plana vitrectomy (PPV) with complete ILM peeling (ILMP) $[11,12]$. However, $\mathrm{MH}$ is brought about in $5-28.6 \%$ of the eyes that underwent PPV with ILMP for MTM [13-29], leading to poor visual prognosis despite reoperation.

In recent years, a new technique of fovea-sparing ILMP, documented by Shimada et al. [24], has been developed to prevent postoperative $\mathrm{MH}$ formation by preserving the foveal Muller cell cone [25]. In the study by Shimada et al. [24], the rate of $\mathrm{MH}$ formation was higher in the complete ILMP group than that in the fovea-sparing ILMP group, although with no statistical significance. In addition, the postoperative best-corrected visual acuity (BCVA) was not significantly better in the fovea-sparing ILMP group. In contrast, in some subsequent studies with larger sample sizes, there was significant difference in postoperative BCVA [25] and central foveal thickness (CFT) [30] between the 2 groups. There have also been more outcomes measured to compare the complete and fovea-sparing ILMP groups. Thus, we conducted this meta-analysis to determine whether the fovea-sparing ILMP improved the anatomic and visual outcomes of MTM in high myopia compared with the complete ILMP technique.

\section{Methods}

The systematic review and meta-analysis were prospectively registered with PROSPERO (https://www.crd.york.ac.uk/PROSPERO/; registration number CRD42020198745). This study was conducted according to the recommendations of the Cochrane Handbook and reported according to the PRISMA Statement.

\section{Search Strategy}

Articles from PubMed, EMBASE, Web of Science, and Cochrane Library were retrieved. All related articles were published before 24 July 2020 in English. We also searched the reference lists of the studies included in the review to identify other eligible studies. The PRISMA 2020 Checklist is presented as online supplemen- tary Table 1 (for all online suppl. material, see www.karger.com/ doi/10.1159/000519021). The following terms for systematic search were used: ("near sight" or nearsighted or myopia or shortsighted or "short sight" or myope or "myopic defocus" or myopic or HM) AND ("inner limiting membrane" OR "internal limiting membrane" OR ILM OR "limiting membrane") AND (peeling OR peel OR removal OR repositioning OR IP OR SIP) AND ("vitreomacular traction" or "retinal thickening" or "macular retinoschisis" or "foveal retinoschisis" or "lamellar macular hole" or MRS or LMH or foveoschisis or "fovea detachment" or "foveal detachment" or "traction maculopathy" or MTM).

\section{Inclusion and Exclusion Criteria}

All publications were screened according to the predefined selection criteria. Inclusion criteria were as follows: (1) patients with MTM; (2) a comparison of the fovea-sparing ILMP and complete ILMP; (3) referred to no less than one of the outcomes indicated; and (4) prospective nonrandomized or randomized control trial or retrospective case series. Exclusion criteria were as follows: (1) a follow-up time $<6$ months; (2) patients with a preoperative fullthickness $\mathrm{MH}$ or macular atrophy that could affect the central vision; (3) abstracts, letters, editorials, and conference proceedings without original data or if from the published results, which was impossible to obtain proper data; and (4) noncomparative studies, single-arm studies, animal studies, case reports, or reviews.

\section{Data Extraction}

Data were independently extracted and evaluated from each included study by 2 reviewers. If the 2 reviewers disagreed, a third reviewer analyzed the data and quality.

The following data were extracted: first author, year of publication, type of trials, country, surgical procedure, number of eyes involved, follow-up time, type of MTM, age, spherical equivalent, axis length (AL), lens status, staining, tamponade, gauge, diameter of the spared foveal area, and measured outcomes. The main outcomes were the rate of development of a postoperative $\mathrm{MH}$ and visual improvement of BCVA (converted to logarithm of the minimum angle of resolution (logMAR). The secondary outcomes were the proportion of patients with visual improvement, the proportion of anatomic success; preoperative and postoperative BCVA, preoperative and postoperative CFT, and time to anatomic resolution.

\section{Qualitative and Risk Bias Assessment}

The prospective nonrandomized comparative study was assessed by the MINORS [31]. Twenty-four is the maximum score for comparative studies. The score of 12 or more indicated a higher quality study. Retrospective studies were assessed by the Newcastle-Ottawa scale, which provided a score from a possible total of 9 stars [32]. Scores of 5 or more indicated that the quality of research was relatively high. Publication bias was assessed using a funnel plot of the data and Egger's test. To assess the possibility of small study bias, we also performed a trim and fill analysis [33].

\section{Statistical Analysis}

Data were analyzed by Review Manager software (V.5.4; The Cochrane Collaboration, Oxford, England). For the dichotomous variables, odds ratios (ORs) and 95\% confidence intervals (CI) were calculated by using Mantel-Haenszel (M-H) method. Continuous variables were compared using weighted mean difference 


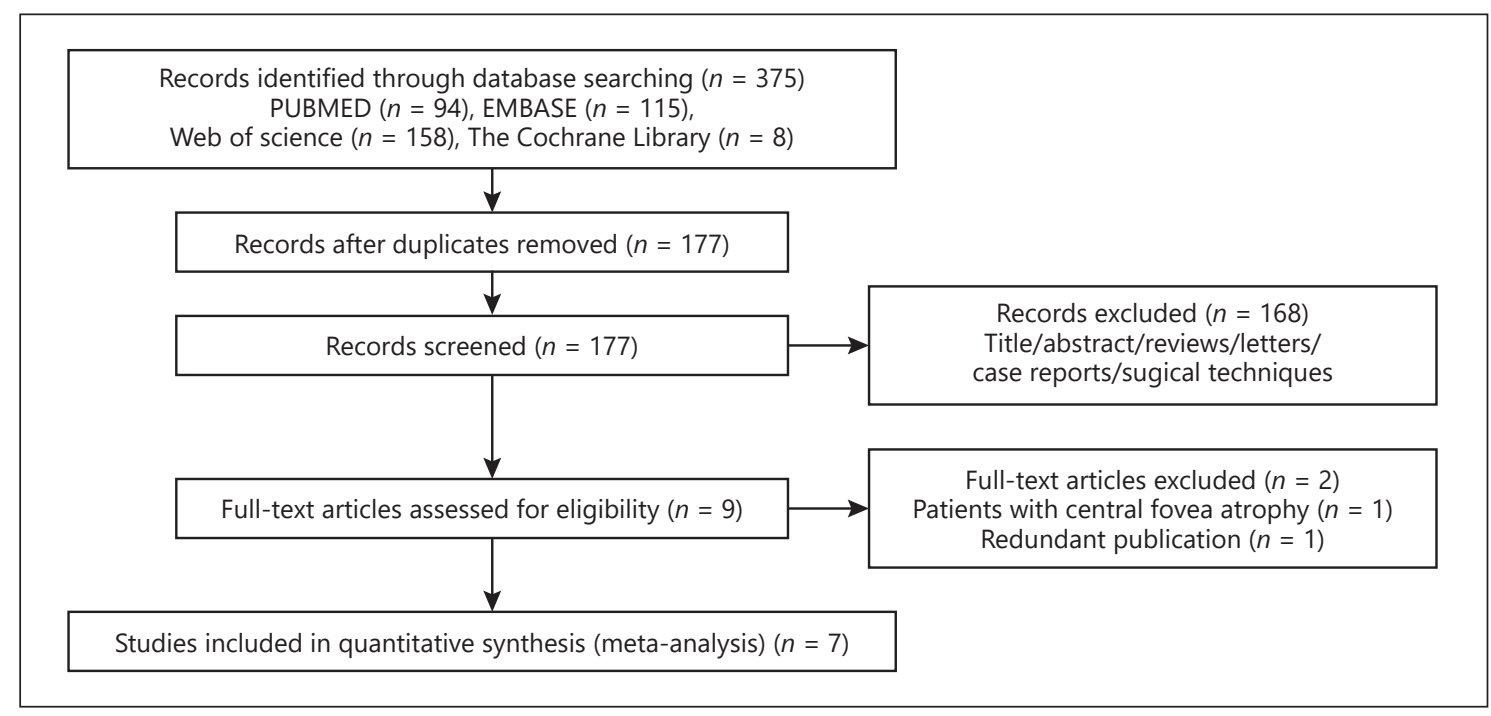

Fig. 1. Flow diagram.

(MD) and 95\% CI. The continuous data such as median and range values in included studies were converted to mean and standard deviation by using the method reported by Hozo et al. [34]. The results of meta-analysis were visually examined by a forest plot. $p$ $<0.05$ was considered statistically significant.

Heterogeneity was assessed by calculating the $I^{2}$ and performing the $\chi^{2}$ test. $I^{2}>50 \%$ and $p<0.05$ suggested considerable heterogeneity. A random-effects model was used for data synthesis in the presence of significant heterogeneity, while a fixed-effects model was used when there was no significant heterogeneity.

In addition, subgroup analyses with follow-up time were used for the study of heterogeneity. A $p$ value $<0.05$ was considered a statistically significant difference between studies. The sensitivity analysis was performed using Stata software (V.15.0; Stata, College Station, TX, USA).

\section{Results}

\section{Selection of Studies}

A total of 375 articles were initially identified through database searching. 177 articles remained after removal of duplicates. After reviewing titles and abstracts, 9 articles were left for full-text assessment. Among the 168 excluded articles, there were 1 book chapter, 2 letters, 2 meta-analyses, 2 conference abstracts, 13 reviews, 16 case reports, 66 articles with irrelevant topics, 25 articles comparing other surgery techniques, and 41 noncomparative articles describing only one kind of surgery technique. Finally, 7 of these studies were eligible for our meta-analysis [24, 25, 30, 35-38] (Fig. 1).

\section{Characteristics of the Included Studies}

Main characteristics of the included studies were presented in Tables 1 and 2. One prospective nonrandomized study [35] and 6 retrospective studies were incorporated. A total of 285 eyes underwent PPV combined with complete ILMP (177 eyes) or fovea-sparing ILMP (108 eyes). The follow-up time of 3 studies were at least 6 months $[24,37,38]$, whereas the other 4 studies were followed up for at least 12 months $[25,30,35,36]$. There was no significant difference in preoperative BCVA (MD -0.01 ; $95 \%$ CI: -0.1 to $0.07 ; p=0.76$; online suppl. Fig. 1a) or preoperative CFT (MD 8.65; 95\% CI: -22.52 to 39.82 ; $p=0.59$; online suppl. Fig. $1 \mathrm{~b}$ ) between the complete and fovea-sparing ILMP group.

\section{Quality Assessment}

The quality assessment of the 7 studies was demonstrated comprehensively in online suppl. Tables 2 and 3. Methodological quality of all the eligible studies was good.

\section{Outcomes}

The Rate of Postoperative MH

The rate of postoperative $\mathrm{MH}$ was $11.86 \%(21 / 177$ eyes) in the complete ILMP group and $0.93 \%$ ( $1 / 108$ eyes) in the fovea-sparing ILMP group. No heterogeneity was observed between the studies ( $p=0.99, I^{2}=0 \%$; Fig. $2 \mathrm{a}$ ). The rate of $\mathrm{MH}$ formation was statistically higher in the complete ILMP group than that in the fovea-sparing ILMP group (OR 5.64; 95\% CI: $1.72-18.44 ; p=0.004$ ). 

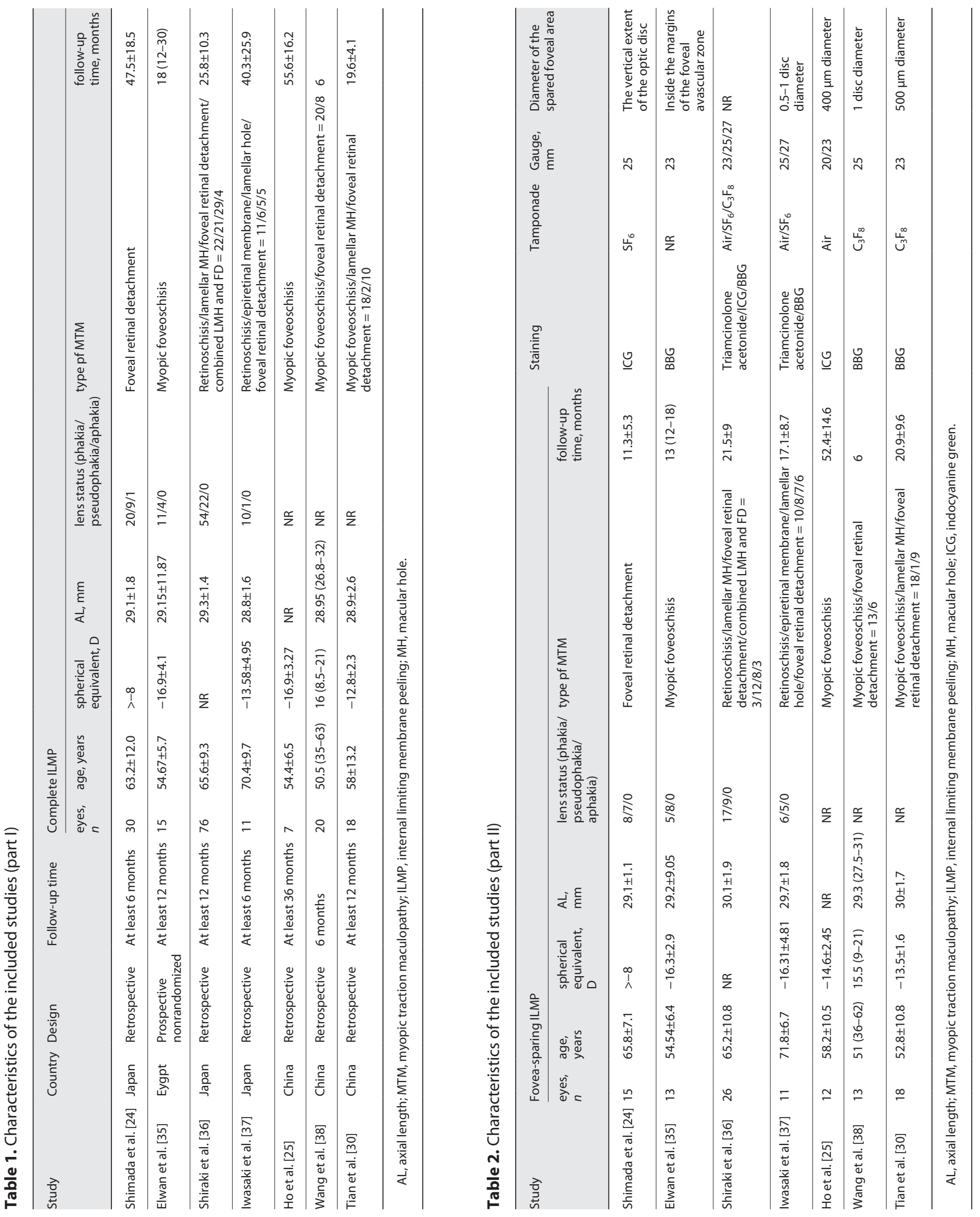


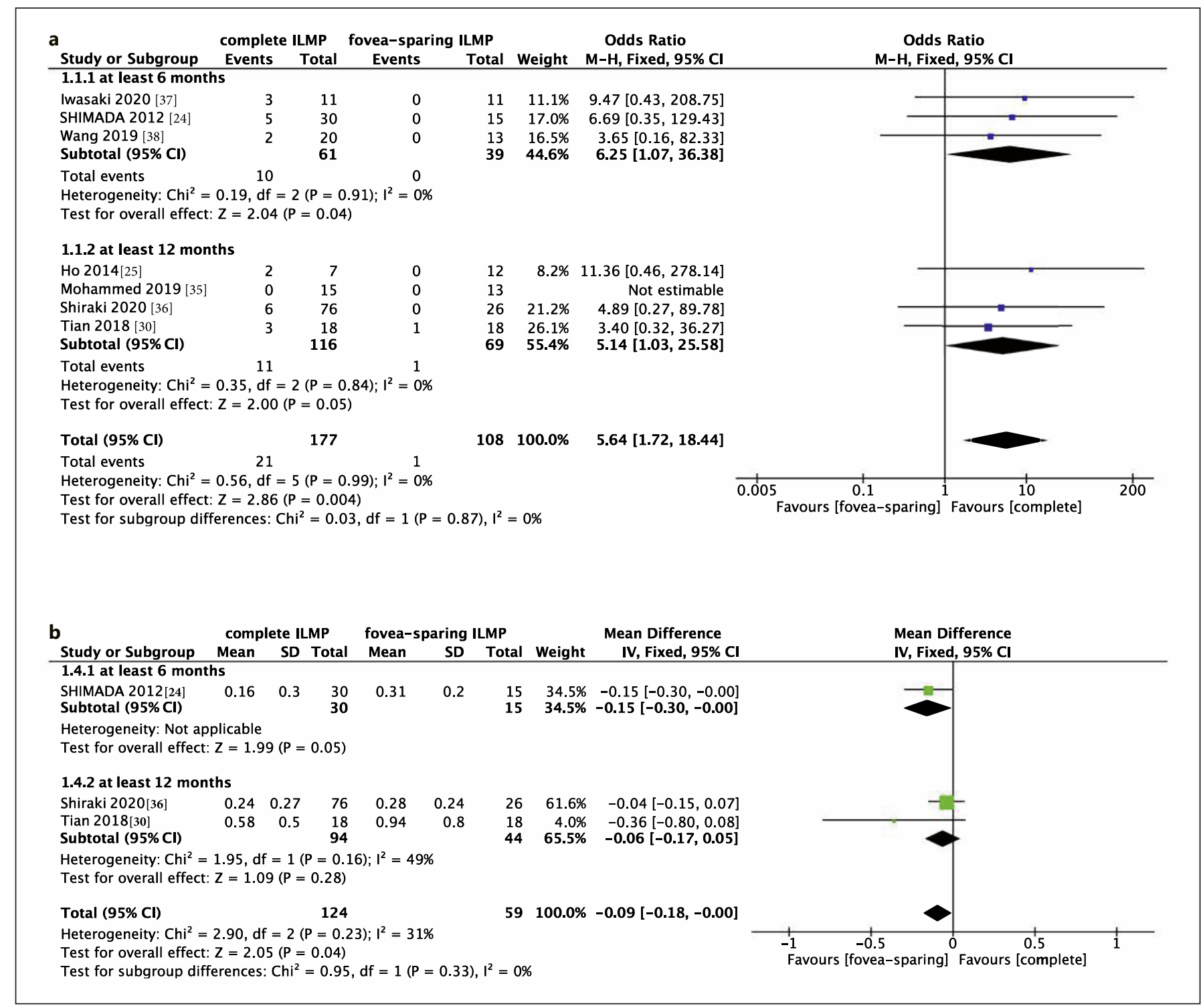

Fig. 2. Forest plot between the fovea-sparing ILMP group and complete ILMP group in MTM. a Forest plot comparing the rate of postoperative $\mathrm{MH}$ formation. b Forest plot comparing the improvement of BCVA in logMAR. ILMP, internal limiting mem-

Postoperative Visual Acuity Analysis

The improvement of BCVA in logMAR was significantly higher in the fovea-sparing ILMP group than that in the complete ILMP group (MD $-0.09 ; 95 \% \mathrm{CI}:-0.18$ to $0.00 ; p=0.04$; Fig. $2 \mathrm{~b}$ ). We found no great heterogeneity among the 3 studies ( $p=0.23, I^{2}=31 \%$ ).

There was considerable heterogeneity among the 7 studies in the postoperative BCVA ( $p=0.02, I^{2}=60 \%$; Fig. 3a). We carried out the subgroup analysis based on follow-up duration that divided the included studies into brane peeling; $\mathrm{MH}$, macular hole; $\mathrm{BCVA}$, best-corrected visual acuity; $\log M A R, \operatorname{logarithm}$ of the minimum angle of resolution; MTM, myopic traction maculopathy; OR, odds ratio; MD, mean difference; CI, confidence interval.

follow-up duration of at least 6 months and at least 12 months. In the subgroup of at least 12 months including 4 studies, the heterogeneity was moderate $\left(p=0.26, I^{2}=\right.$ $25 \%)$. Postoperative BCVA was not significantly different between the fovea-sparing ILMP and complete ILMP groups (MD 0.14; 95\% CI: 0.00-0.27; $p=0.05$ ). Heterogeneity was still high among the remaining 3 studies in the subgroup of follow-up duration for at least 6 months (heterogeneity $I^{2}=64 \%$ ). A random-effects model was used in the subgroup analysis. 
a

complete ILMP fovea-sparing ILMP

Study or Subgroup Mean SD Total Mean SD Total Weight IV, Random, $95 \% \mathrm{CI}$

1.6.1 at least 6 months

Iwasaki 2020[37]

SHIMADA 2012[24]

Wang 2019 [38]

0.520 .43

$\begin{array}{lll}11 & 0.34 & 0.42\end{array}$

$11 \quad 9.0 \%$

$0.18[-0.18,0.54]$

Subtotal $(95 \% \mathrm{Cl})$

$\begin{array}{rrrrr}0.58 & 0.6 & 30 & 0.34 & 0.4\end{array}$

$15 \quad 11.3 \%$

$0.07[-0.06,0.54]$

$39-44.7 \%-0.07[-0.16,0.01]$

Heterogeneity: $\mathrm{Tau}^{2}=0.03 ; \mathrm{Chi}^{2}=5.59, \mathrm{df}=2(\mathrm{P}=0.06) ; \mathrm{I}^{2}=64 \%$

Test for overall effect: $Z=0.62(P=0.54)$

1.6.2 at least 12 months

$\begin{array}{lrrrrrrrr}\text { Ho 2014[25] } & 1.39 & 0.33 & 7 & 0.89 & 0.56 & 12 & 7.6 \% & 0.50[0.10,0.90] \\ \text { Mohammed 2019[35] } & 0.83 & 0.23 & 15 & 0.7 & 0.26 & 13 & 17.7 \% & 0.13[-0.05,0.31] \\ \text { Shiraki 2020[36] } & 0.37 & 0.38 & 76 & 0.32 & 0.43 & 26 & 17.5 \% & 0.05[-0.14,0.24] \\ \text { Tian 2018[30] } & 0.67 & 0.5 & 18 & 0.56 & 0.3 & 18 & 12.6 \% & 0.11[-0.16,0.38] \\ \text { Subtotal (95\% CI) } & & & \mathbf{1 1 6} & & & \mathbf{6 9} & \mathbf{5 5 . 3 \%} & \mathbf{0 . 1 4}[\mathbf{0 . 0 0} \mathbf{0 . 2 7}]\end{array}$

Subtotal $(95 \% \mathrm{Cl})$

Heterogeneity: $\mathrm{Tau}^{2}=0.00 ; \mathrm{Chi}^{2}=4.01, \mathrm{df}=3(\mathrm{P}=0.26) ; \mathrm{I}^{2}=25 \%$

Test for overall effect: $Z=1.97(P=0.05)$

$\begin{array}{lllll}\text { Total }(95 \% \mathrm{Cl}) & 177 & 108 & 100.0 \% & 0.11[-0.02,0.24]\end{array}$

Heterogeneity: $\mathrm{Tau}^{2}=0.02 ; \mathrm{Chi}^{2}=15.16, \mathrm{df}=6(\mathrm{P}=0.02) ; \mathrm{I}^{2}=60 \%$

Test for overall effect: $Z=1.64(P=0.10)$

Test for subgroup differences: $\mathrm{Chi}^{2}=0.22, \mathrm{df}=1(\mathrm{P}=0.64), \mathrm{I}^{2}=0 \%$

Complete ILMP fovea-sparing ILMP Odds Ratio

Study or Subgroup Events Total Events Total Weight $\mathrm{M}-\mathrm{H}$, Random, $95 \% \mathrm{Cl}$ 1.2.1 at least 6 months SHIMADA $2012[\mathbf{2 4}]$ Subtotal $(95 \% \mathrm{Cl})$

$\begin{array}{rrr}4 & 11 \\ 16 & 30\end{array}$

11
30
41

7
11

$1120.9 \%$

$15 \quad 28.5 \%$

$0.33[0.06,1.86]$

Total events 20

$20 \quad 18$

Heterogeneity: $\mathrm{Tau}^{2}=0.00 ; \mathrm{Chi}^{2}=0.05, \mathrm{df}=1(\mathrm{P}=0.83) ; \mathrm{I}^{2}=0 \%$

Test for overall effect: $Z=1.78(P=0.07)$

1.2.2 at least 12 months

$\begin{array}{lrrrrr}\text { Shiraki } 2020[36] & 32 & 76 & 13 & 26 & 41.4 \% \\ \text { Tian 2018 [30] } & 9 & 18 & 18 & 18 & 9.2 \% \\ \text { Subtotal }(95 \% \mathrm{Cl}) & & 94 & & 44 & \mathbf{5 0 . 6 \%}\end{array}$

Subtotal $(95 \% \mathrm{Cl})$

41 31

$0.42[0.11,1.60]$
$0.38[0.13,1.10]$

Heterogeneity: $\mathrm{Tau}^{2}=4.75 ; \mathrm{Chi}^{2}=4.84, \mathrm{df}=1(\mathrm{P}=0.03) ; \mathrm{I}^{2}=79 \%$

Test for overall effect: $Z=0.99(P=0.32)$

$\begin{array}{lllll}\text { Total }(95 \% \mathrm{Cl}) & 135 & 70 & 100.0 \% & 0.39[0.15,1.02\end{array}$

Total events

61

49

Heterogeneity: $\mathrm{Tau}^{2}=0.38 ; \mathrm{Chi}^{2}=4.98, \mathrm{df}=3(\mathrm{P}=0.17) ; \mathrm{I}^{2}=40 \%$

Test for overall effect: $Z=1.92(P=0.05)$

Test for subgroup differences: $\mathrm{Chi}^{2}=0.16, \mathrm{df}=1(\mathrm{P}=0.69), \mathrm{I}^{2}=0 \%$

C complete ILMP fovea-sparing ILMP

Study or Subgroup Mean SD Total Mean SD Total Weight

1.8.1 at least 6 months

$\begin{array}{llllllll}\text { Iwasaki 2020 [37] } & 130.3 & 38 & 11 & 128.8 & 46.5 & 11 & 16.4 \%\end{array}$

$\begin{array}{rrrrr}130.3 & 38 & 11 & 128.8 & 46.5 \\ 317 & 42.5 & 20 & 311.25 & 65.553\end{array}$

Wang $2019[38]$
Subtotal (95\% C $)$

31

$\begin{array}{ll}11 & 16.4 \% \\ 13 & 12.8 \%\end{array}$

$\begin{array}{ll}13 & 12.8 \% \\ 24 & 29.2 \%\end{array}$

Heterogeneity: $\mathrm{Chi}^{2}=0.02, \mathrm{df}=1(\mathrm{P}=0.88) ; \mathrm{I}^{2}=0 \%$

Test for overall effect: $Z=0.25(P=0.80)$

1.8.2 at least 12 months

$\begin{array}{lllllllll}\text { Ho } 2014[25] & 122 & 67 & 7 & 137 & 52 & 12 & 6.2 \% & -15.00[-72.70,42.70]\end{array}$

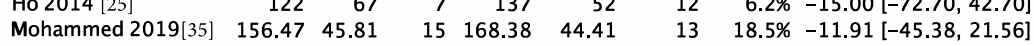

$\begin{array}{lrrrrrrrr}\text { Mohammed 2019[35] } & 156.47 & 45.81 & 15 & 168.38 & 44.41 & 13 & 18.5 \% & -11.91[-45.38,21.56] \\ \text { Shiraki 2020 [36] } & 128.4 & 51.7 & 76 & 148.9 & 52.9 & 26 & 37.7 \% & -20.50[-43.92,2.92]\end{array}$

$\begin{array}{llllll}\text { Tian } 2018[30] & 141.6 & 93.4 & 18 & 143.36 & 52.4\end{array}$

Subtotal $(95 \% \mathrm{Cl})$

116

$18 \quad 8.4 \% \quad-1.76[-51.23,47.71]$

Heterogeneity: $\mathrm{Chi}^{2}=0.52, \mathrm{df}=3(\mathrm{P}=0.92) ; \mathrm{I}^{2}=0 \%$

Test for overall effect: $Z=1.78(P=0.07)$

Total $(95 \% \mathrm{Cl})$

147

$93 \quad 100.0 \%-10.02[-24.40,4.36]$

Heterogeneity: $\mathrm{Chi}^{2}=1.91, \mathrm{df}=5(\mathrm{P}=0.86) ; \mathrm{I}^{2}=0 \%$

Test for overall effect: $Z=1.37(P=0.17)$

Test for subgroup differences: $\mathrm{Chi}^{2}=1.37, \mathrm{df}=1(\mathrm{P}=0.24), \mathrm{I}^{2}=27.2 \%$

$0.73[0.30,1.78]$

$0.03[0.00,0.52]$
$0.19[0.01,5.25]$

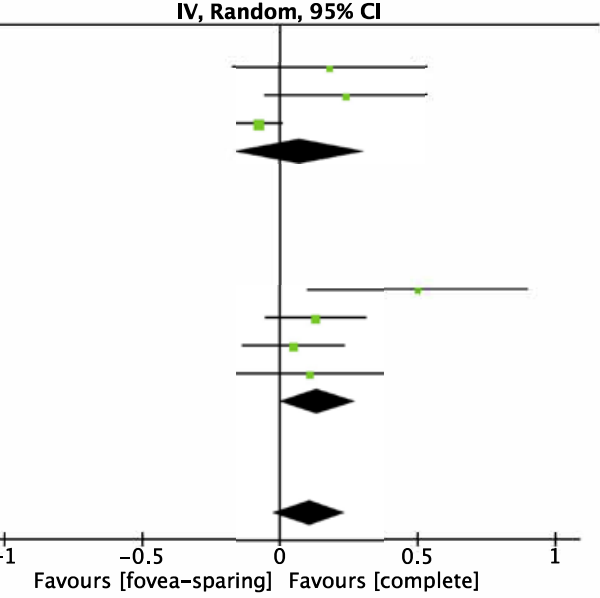

3

(For legend see next page.)

Internal Limiting Membrane Peeling for Myopic Traction Maculopathy
Ophthalmic Res 2021;64:916-927

DOI: $10.1159 / 000519021$ M-H, Random, 95\% Cl

IV, Random, $95 \%$ C

Odds Ratio

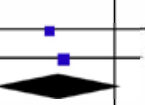

Favours [fovea-sparing] Favours [complete] 100

Mean Difference IV, Fixed, 95\% CI

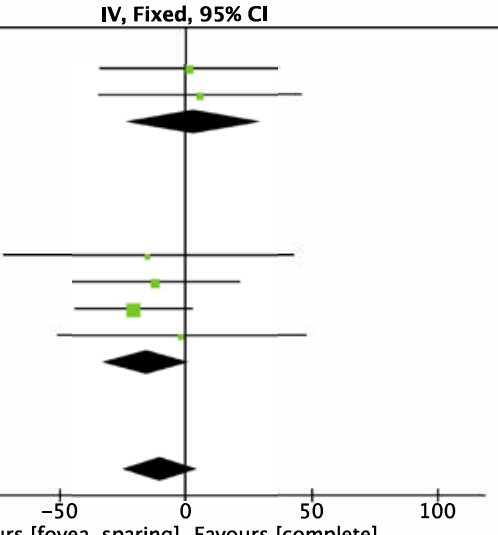

Favours [fovea-sparing] Favours [complete]

921 


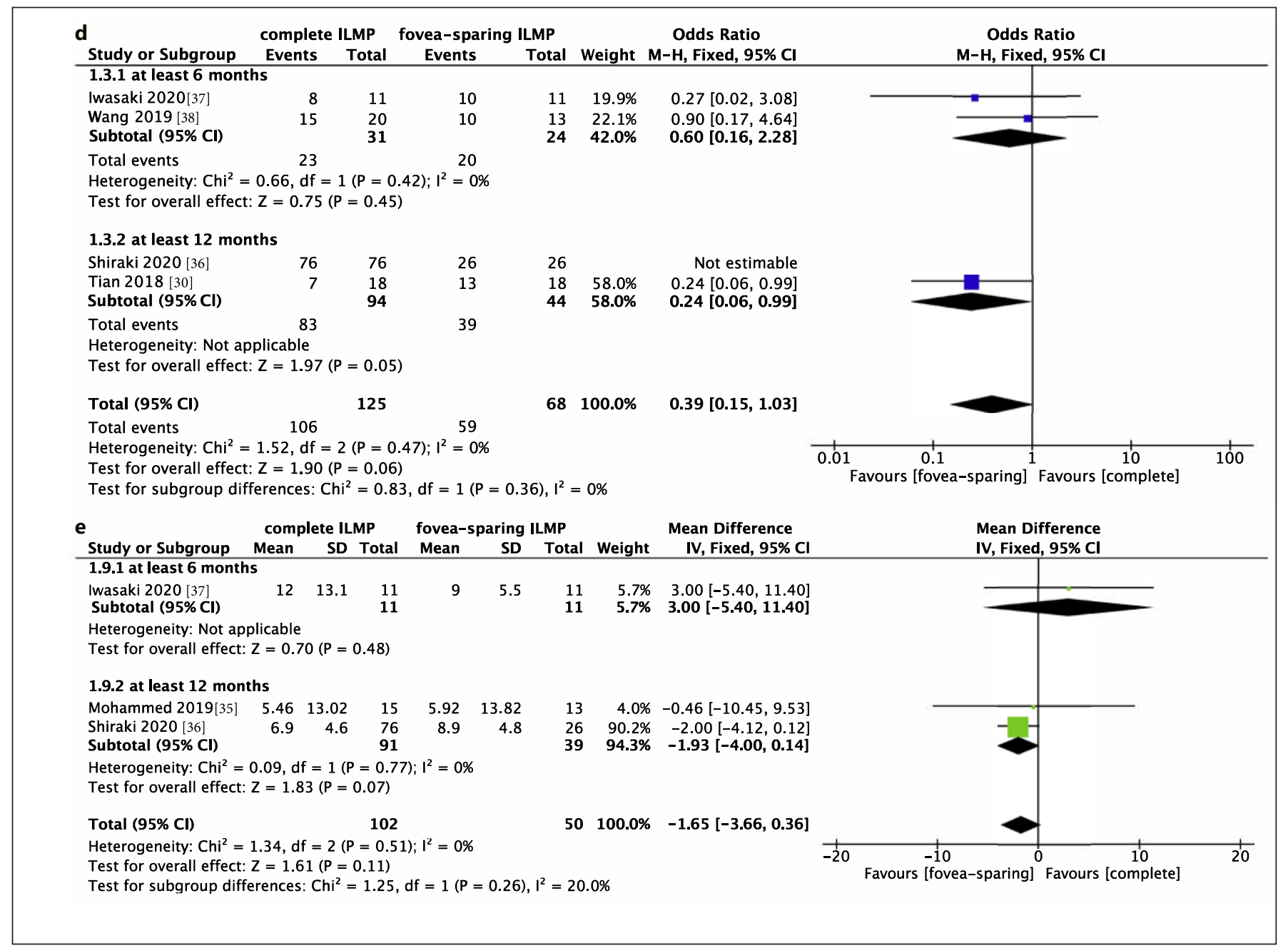

Fig. 3. Forest plot between the fovea-sparing ILMP group and complete ILMP group in MTM. a Forest plot comparing the postoperative BCVA. b Forest plot comparing the proportion of patients with visual improvement. c Forest plot comparing the postoperative CFT. d Forest plot comparing the proportion of ana-

There was no significant difference in the proportion of patients with visual improvement between the complete ILMP and fovea-sparing ILMP groups (OR 0.39; 95\% CI: $0.15-1.02 ; p=0.05)$. However, the heterogeneity among the 4 studies was moderate $\left(p=0.17, I^{2}=40 \%\right.$, Fig. 3b). In the subgroup of follow-up time for at least 12 months, the heterogeneity was relatively high $(p=0.03$, $\left.I^{2}=79 \%\right)$, while no heterogeneity was observed in the subgroup of a shorter follow-up time $\left(p=0.83, I^{2}=0 \%\right)$.

\section{Postoperative CFT}

The postoperative CFT using fovea-sparing ILMP was not statistically different from that using complete ILMP tomic success. e Forest plot comparing the time to resolution. CFT, central fovea thickness; ILMP, internal limiting membrane peeling; BCVA, best-corrected visual acuity; MTM, myopic traction maculopathy; OR, odds ratio; $\mathrm{MD}$, mean difference; CI, confidence interval.
(MD -10.02; 95\% CI: -24.4 to $4.36 ; p=0.17$; Fig. $3 c$ ). No heterogeneity was observed among these 6 studies $(p=$ $\left.0.86, I^{2}=0 \%\right)$.

\section{Anatomic Success Analysis}

There was a tendency of anatomic success of the foveasparing ILMP group compared with that of the complete ILMP group, although with no statistical significance (MD 0.39; 95\% CI: 0.15-1.03; $p=0.06$; Fig. 3d). In addition, no significance was found in the time to resolution between the 2 groups ( $\mathrm{MD}-1.65$; $95 \% \mathrm{CI}:-3.66$ to 0.36 ; $p=0.11$; Fig. 3e). 


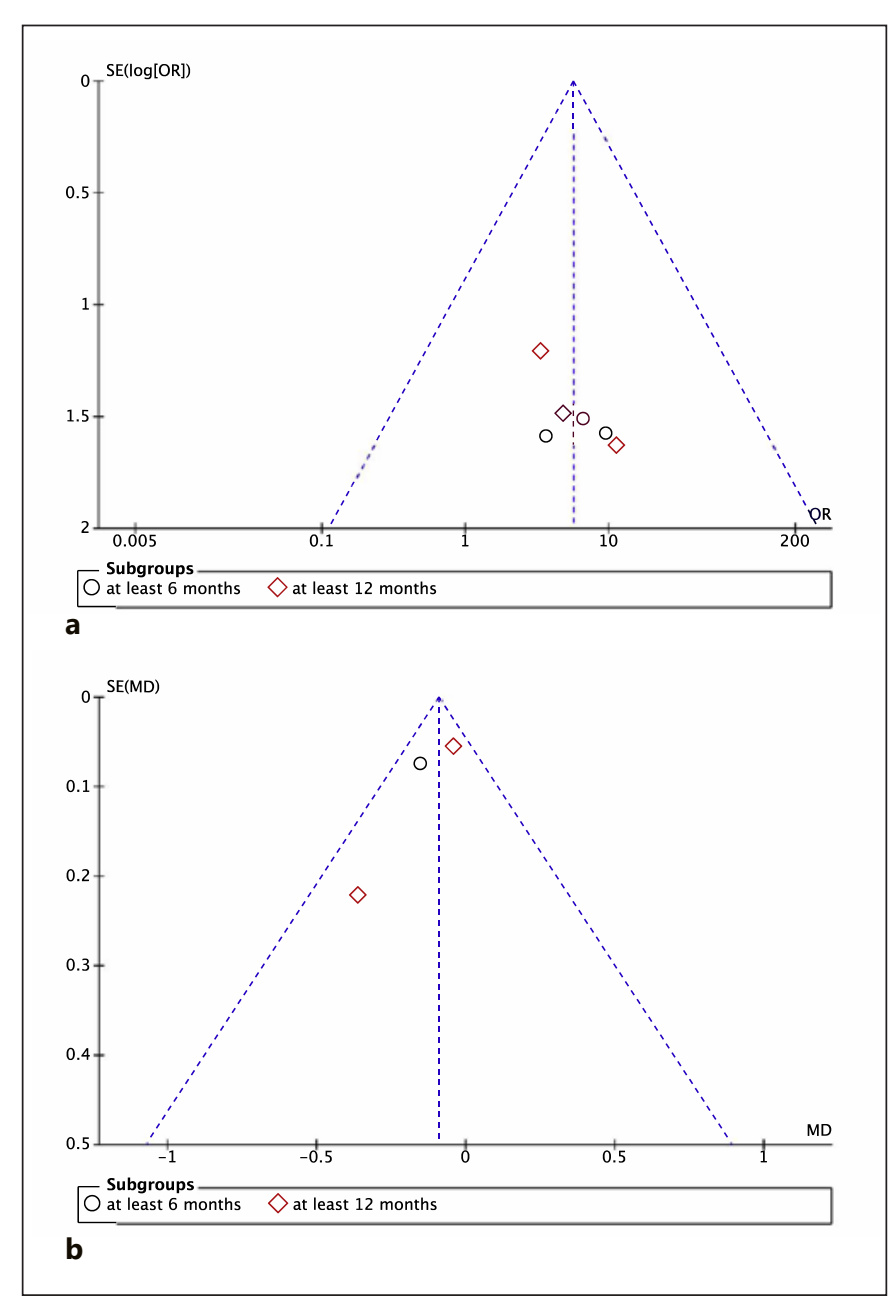

Fig. 4. Funnel plot analysis of main outcomes: the rate of postoperative $\mathrm{MH}$ formation (a) and the improvement of BCVA in logMAR (b). SE, standard error; OR, odds ratio; MD, mean difference; $\mathrm{CI}$, confidence interval; $\mathrm{MH}$, macular hole; BCVA, best-corrected visual acuity; $\log \mathrm{MAR}$, logarithm of the minimum angle of resolution.

\section{Publication Bias}

Funnel plots of the rate of postoperative $\mathrm{MH}$ (Fig. 4a), preoperative BCVA, the improvement of BCVA in logMAR (Fig. 4b), preoperative and postoperative CFT, the rate of anatomic success, and time to resolution showed that the scattered points of the included studies were mainly distributed in the middle and top of the baseline, and the points were located in the range of inverted funnel. It suggested that there was no serious publication bias and the conclusion is relatively reliable. However, there was bias in the postoperative BCVA ( $p=0.002$, online suppl. Fig. 2a) and the proportion of patients with visual improvement ( $p=0.027$, online suppl. Fig. $2 b$ ). After us- ing the trim and fill, and adjusting the effect size for funnel plot asymmetry, the adjusted effect was the same to the original effect (online suppl. Fig. 3). For the postoperative BCVA and the proportion of patients with visual improvement, the ORs after trim-and-fill method were 0.03 (95\% CI: -0.09 to $0.15 ; p=0.598)$ and 0.59 (95\% CI: $0.222-1.577 ; p=0.294)$.

\section{Sensitivity Analysis}

Outcomes of sensitivity analysis were demonstrated in online suppl. Figure 4. Studies on all the measured outcomes, except for the time to resolution had good stability. The sensitivity analysis of the time to resolution indicated that the result varied whether this study was included [36]. When this study was excluded, the MD was 1.57 , with the $95 \%$ CI of -4.86 to 8.00 . After including it, the MD was -1.65 , with the $95 \%$ CI of -3.66 to 0.36 .

\section{Discussion}

To date, our meta-analysis was the first registered one to compare the anatomic and visual outcomes of PPV with fovea-sparing ILMP versus complete ILMP for MTM. Seven comparative studies with a total of 285 eyes were reviewed. Pooled results indicated that there was a lower rate of postoperative $\mathrm{MH}$ formation and more improvement of BCVA in logMAR in fovea-sparing ILMP group. However, postoperative BCVA, the proportion of patients with visual improvement, postoperative CFT, the rate of anatomic success, and time to resolution showed no significant differences.

Different surgical approaches for conserving the epifoveal ILM have been published in various studies. Some previous studies [24, 30, 35-37] applied the technique, which was named as "fovea-sparing ILMP." Ho et al. [25] reported the use of micro-scissors to induce numerous tangential cuts in the ILM around the fovea and it was referred to as "the fovea nonpeeling technique." Wang et al. [38] described the technique to preserve the central fovea as "partial ILMP to preserve the ILM of the central fovea."

The rate of postoperative $\mathrm{MH}$ formation was significantly lower in the fovea-sparing ILMP group than that in the complete ILMP group. In 6 of all the 7 studies, nobody in the fovea-sparing ILMP group developed a postoperative $\mathrm{MH}$. The pathogenesis of $\mathrm{MH}$ after vitrectomy for MTM remains unclear. It was hypothesized that the Muller cells endfeet within the preserved ILM 
may contribute to maintaining the foveolar cone skeleton [39]. The loss of Muller cell cone in the fovea and the weakening of macular glial structure may result in the development of a $\mathrm{MH}$ [20]. The integrity of fine processes of the Muller cells that envelop all the neurons is maintained by the fovea-sparing ILMP technique. For patients who underwent fovea-sparing ILMP, the release of macular traction and less surgical trauma to the central fovea caused a centripetal contraction of the remaining ILM, which likely explained why a full-thickness MH did not develop. However, it was also proposed that some types of MTM are extremely weak and can be more easily damaged, regardless of whether it is managed with fovea-sparing or total ILMP, such as outer lamellar MH [30].

With regard to visual prognosis, both groups presented with certain improvement of BCVA in $\log M A R$, referred to the BCVA gain after operation. It was found that the improvement of BCVA was statistically higher in the fovea-sparing ILMP group. The proportion of patients with improved BCVA was higher in the fovea-sparing ILMP group, although with no significant difference. Considering the high proportion of patients with postoperative MHs in complete ILMP group, we speculated that the absence of a postoperative $\mathrm{MH}$ could contribute to the better improvement of BCVA in patients who underwent the fovea-sparing ILMP. Additionally, the follow-up time was longer in the complete ILMP group, chorioretinal atrophy in MTM may progress and lead to the worsening of VA in such a long period [40]. Moreover, greater CFT was associated with favorable visual outcomes. It could be explained by the fact that there was less chorioretinal atrophy at the fovea in patients with a greater fovea thickness. The postoperative CFT was higher in the foveasparing ILMP group, especially in the subgroup with a follow-up time of $>12$ months. We supposed that a shorter follow-up time may result in incomplete repair of the macular structure, thus leading to insufficient recovery of visual function.

In contrast to expectations, there was no significant difference in postoperative BCVA between the complete ILMP group and fovea-sparing ILMP group. Considering the heterogeneity in postoperative BCVA among these studies, we divided them into 2 subgroups based on follow-up duration to explore the short and longterm visual efficacy. At a follow-up of $>12$ months, postoperative BCVA was better in the group of fovea-sparing ILMP compared with complete ILMP, with no significant difference. It seemed that patients who underwent fovea-sparing ILMP tended to have a visual gain in the long term. For patients accompanied by foveal detachment or with high $\mathrm{AL}$, the preoperative and postoperative BCVAs were poorer [38]. There were only 2 studies in which no patients with foveal detachment were included $[25,35]$. On the one hand, the foveal photoreceptor layer may have been destroyed before surgery, and the foveal structure of the outer retinal layer might not recover. Nevertheless, there is also some concern that a late contraction of the remaining ILM on the fovea may lead to visual function reduction by a mechanism similar to that of epiretinal membrane formation [24]. Fovea-sparing ILMP for MTM and its long-term course should be considered carefully [37]. To reduce the contraction of the residual ILM in the fovea, Lee et al. [41] improved ILMP surgical procedure to further reduce the area of the remaining ILM. In the process of fovea-sparing ILMP, various diameters of fovea were spared in different studies. There is no consensus on the criteria for this surgical procedure to treat MTM with high myopia. The difficulty of fovea-sparing ILMP should be taken into account when choosing ILMP technique. In addition, the effect of surgical learning curve of sparing the fovea cannot be completely ruled out [24]. For eyes with long ALs, chorioretinal atrophy and posterior scleral staphyloma, it is extremely challenging to peel the ILM, especially preserve the epi-foveal ILM [30]. ILM of eyes with high myopia is thin and difficult to identify, which may lead to iatrogenic injury, such as holes or retinal detachment. Unresolving schisis and/or $\mathrm{MH}$ formation might occur if the ILM is left at a location other than the fovea. Persistent or later recurrent foveoschisis may occur after vitrectomy with incomplete ILM peeling, due to fibrocellular proliferation on the residual vitreous cortex or residual ILM over the posterior pole [42]. We ought to be fully aware of the difficulty and risk of fovea-sparing ILMP.

In addition, correction for potential publication bias did not alter the insignificant association between the postoperative BCVA and the proportion of patients with visual improvement with the fovea-sparing ILMP technique. The outcomes would be even more stable and reliable with supplement of more studies.

Other anatomic outcomes such as time to resolution and postoperative CFT did not differ statistically between the 2 groups. It indicated that the sparing of the fovea would not hinder the anatomic recovery of MTM. In the meantime, the remaining ILM at the spared fovea did not interfere with the process of MTM resolution by a traction force [36]. Nevertheless, the sensitivity analysis of time to resolution revealed that this study [36] affected 
the results a lot, which meant that the studies on the time to resolution was unstable. The discrepancy of MTM type in the 3 studies might account for this outcome in sensitivity analysis for time to resolution. Additionally, the sample size of the study [36] was much larger than that of the other 2 studies. The type of staining might also explain for the instability. In the study, some of patients received staining of indocyanine green, which was not used in the other 2 studies.

There is still controversy over the necessity and category of the intraocular tamponade filling. Gas remains only for 1 or 2 months, while the resolution of schisis requires more prolonged time. A meta-analysis reported that gas tamponade had no significant impact neither on the resolution of MF or on the visual acuity [43]. Some researchers have recommended that it is not obligatory to use tamponade as long as no full-thickness hole is detected $[23,44]$. Different tamponades have been used, including C3F8, SF6, air, etc. Compared with gas, air has a shorter absorption time in the eye. It has been reported that air tamponade is comparable to long-effect gas filling like C3F8 or SF6 in the treatment of MH [45-49]. More studies could be conducted to compare the efficacy of no tamponade, air, gas, and other tamponades in the surgical process for MTM.

There were several limitations that should be taken into consideration when interpreting the results of our meta-analysis. First, the number of included studies and available data were limited, resulting in the lack of additional information of treatment for MTM. Second, 6 of all the 7 studies for the meta-analysis were retrospective. Third, there was significant heterogeneity among the studies in comparison of postoperative BCVA, which was alleviated but not eliminated completely through subgroup analysis. Follow-up durations in some of the included trials were not long enough for better observations of VA recovery in the long run. Other influence factors, such as dye, tamponade, retinal nerve fiber layer thickness, and the diameter of spared fovea among the studies might serve as the points of heterogeneity. Due to the deficiency of these available data, we did not perform related subgroup analysis. Fourth, converting non-normally distributed statistics (median and range) to normally distributed statistics (mean and SD) and publication bias may be a cause of bias, despite that the trim and fill method showed the adjusted effect was the same with the original effect. Additionally, the sensitivity analysis of the time to resolution indicated that the result depended on the inclusion of one study [36].

Internal Limiting Membrane Peeling for Myopic Traction Maculopathy
In conclusion, the fovea-sparing ILMP could contribute to a lower $\mathrm{MH}$ formation rate and more improvement of BCVA in logMAR than complete ILMP. More and larger randomized and prospective studies with longer follow-up duration are necessary to further confirm the effects of the fovea-sparing technique for MTM. It was also an innovative need to provide improved and standardized surgical indications and methods for better safety and efficacy for MTM.

\section{Statement of Ethics}

All analyses were based on published studies, and thus, no ethical approval and informed consent are required. Nonetheless, the study adhered fully to the Declaration of Helsinki.

\section{Conflict of Interest Statement}

The authors have no conflicts of interest to declare.

\section{Funding Sources}

This work was supported by the National Key R\&D Program of China (No. 2020YFC2008200).

\section{Author Contributions}

Study design and concept were developed by Qiaozhu Zeng, Yuou Yao, and Mingwei Zhao; database search was performed by Qiaozhu Zeng, Yuou Yao, and Mingwei Zhao; data extracting was performed by Qiaozhu Zeng, Yuou Yao, and Mingwei Zhao; data analysis was performed by Qiaozhu Zeng and Yuou Yao; and the manuscript was written by Qiaozhu Zeng.

\section{Data Availability Statement}

All data generated or analyzed during this study are included in this article or its online suppl. files. Further inquiries can be directed to the corresponding author. 


\section{References}

1 Phillips CI. Retinal detachment at the posterior pole. Br J Ophthalmol. 1958;42:749-53.

2 Takano M, Kishi S. Foveal retinoschisis and retinal detachment in severely myopic eyes with posterior staphyloma. Am J Ophthalmol. 1999;128:472-6.

3 Panozzo G, Mercanti A. Optical coherence tomography findings in myopic traction maculopathy. Arch Ophthalmol. 2004;122: 1455-60.

4 Baba T, Ohno-Matsui K, Futagami S, Yoshida T, Yasuzumi K, Kojima A, et al. Prevalence and characteristics of foveal retinal detachment without macular hole in high myopia. Am J Ophthalmol. 2003;135:338-42.

5 Shimada N, Ohno-Matsui K, Baba T, Futagami S, Tokoro T, Mochizuki M. Natural course of macular retinoschisis in highly myopic eyes without macular hole or retinal detachment. Am J Ophthalmol. 2006;142: 497-500.

6 Gaucher D, Haouchine B, Tadayoni R, Massin P, Erginay A, Benhamou N, et al. Longterm follow-up of high myopic foveoschisis: natural course and surgical outcome. Am J Ophthalmol. 2007;143:455-62.

7 Shimada N, Tanaka Y, Tokoro T, OhnoMatsui K. Natural course of myopic traction maculopathy and factors associated with progression or resolution. Am J Ophthalmol. 2013;156:948-57.e1.

8 Benhamou N, Massin P, Haouchine B, Erginay A, Gaudric A. Macular retinoschisis in highly myopic eyes. Am J Ophthalmol. 2002;133:794-800.

9 Sayanagi K, Ikuno Y, Tano Y. Tractional internal limiting membrane detachment in highly myopic eyes. Am J Ophthalmol. 2006; 142(5):850-2.

10 Bando H, Ikuno Y, Choi JS, Tano Y, Yamanaka I, Ishibashi T. Ultrastructure of internal limiting membrane in myopic foveoschisis. Am J Ophthalmol. 2005;139:197-9.

11 Johnson MW. Myopic traction maculopathy: pathogenic mechanisms and surgical treatment. Retina. 2012;32 Suppl 2:S205-10.

12 VanderBeek BL, Johnson MW. The diversity of traction mechanisms in myopic traction maculopathy. Am J Ophthalmol. 2012; 153:93-102.

13 Shin JY, Yu HG. Visual prognosis and spectral-domain optical coherence tomography findings of myopic foveoschisis surgery using 25-gauge transconjunctival sutureless vitrectomy. Retina. 2012;32:486-92.

14 Hattori K, Kataoka K, Takeuchi J, Ito Y, Terasaki $\mathrm{H}$. Predictive factors of surgical outcomes in vitrectomy for myopic traction maculopathy. Retina. 2018;38 Suppl 1:S2330.

15 Taniuchi S, Hirakata A, Itoh Y, Hirota K, Inoue M. Vitrectomy with or without internal limiting membrane peeling for each stage of myopic traction maculopathy. Retina. 2013;33:2018-25.
16 Figueroa MS, Ruiz-Moreno JM, Gonzalez del Valle F, Govetto A, de la Vega C, Plascencia RN, et al. Long-term outcomes of 23-gauge pars plana vitrectomy with internal limiting membrane peeling and gas tamponade for myopic traction maculopathy: a prospective study. Retina. 2015;35(9):183643.

17 Hwang JU, Joe SG, Lee JY, Kim JG, Yoon $\mathrm{YH}$. Microincision vitrectomy surgery for myopic foveoschisis. Br J Ophthalmol. 2013; 97:879-84.

18 Ikuno Y, Sayanagi K, Soga K, Oshima Y, Ohji M, Tano Y. Foveal anatomical status and surgical results in vitrectomy for myopic foveoschisis. Jpn J Ophthalmol. 2008;52: 269-76.

19 Kumagai K, Furukawa M, Ogino N, Larson E. Factors correlated with postoperative visual acuity after vitrectomy and internal limiting membrane peeling for myopic foveoschisis. Retina. 2010;30:874-80.

20 Gao X, Ikuno Y, Fujimoto S, Nishida K. Risk factors for development of full-thickness macular holes after pars plana vitrectomy for myopic foveoschisis. Am J Ophthalmol. 2013;155:1021-7.e1.

21 Panozzo G, Mercanti A. Vitrectomy for myopic traction maculopathy. Arch Ophthalmol. 2007;125:767-72.

22 Lim SJ, Kwon YH, Kim SH, You YS, Kwon OW. Vitrectomy and internal limiting membrane peeling without gas tamponade for myopic foveoschisis. Graefes Arch Clin Exp Ophthalmol. 2012;250:1573-7.

23 Kim KS, Lee SB, Lee WK. Vitrectomy and internal limiting membrane peeling with and without gas tamponade for myopic foveoschisis. Am J Ophthalmol. 2012;153: 320-6.e1.

24 Shimada N, Sugamoto Y, Ogawa M, Takase $\mathrm{H}$, Ohno-Matsui K. Fovea-sparing internal limiting membrane peeling for myopic traction maculopathy. Am J Ophthalmol. 2012; 154:693-701.

25 Ho TC, Yang CM, Huang JS, Yang CH, Yeh PT, Chen TC, et al. Long-term outcome of foveolar internal limiting membrane nonpeeling for myopic traction maculopathy. Retina. 2014;34:1833-40.

26 Uchida A, Shinoda H, Koto T, Mochimaru H, Nagai N, Tsubota K, et al. Vitrectomy for myopic foveoschisis with internal limiting membrane peeling and no gas tamponade. Retina. 2014;34:455-60.

27 Kobayashi H, Kishi S. Vitreous surgery for highly myopic eyes with foveal detachment and retinoschisis. Ophthalmology. 2003; 110:1702-7.

28 Futagami S, Inoue M, Hirakata A. Removal of internal limiting membrane for recurrent myopic traction maculopathy. Clin Exp Ophthalmol. 2008;36:782-5.
29 Zheng B, Chen Y, Chen Y, Zhao Z, Zhang Z, Zheng J, et al. Vitrectomy and internal limiting membrane peeling with perfluoropropane tamponade or balanced saline solution for myopic foveoschisis. Retina. 2011;31: 692-701.

30 Tian T, Jin H, Zhang Q, Zhang X, Zhang H, Zhao P. Long-term surgical outcomes of multiple parfoveolar curvilinear internal limiting membrane peeling for myopic foveoschisis. Eye. 2018;32:1783-9.

31 Slim K, Nini E, Forestier D, Kwiatkowski F, Panis Y, Chipponi J. Methodological index for non-randomized studies (minors): development and validation of a new instrument. ANZ J Surg. 2003;73:712-6.

32 Wells GA, Shea B, O'Connell D, Peterson J, Welch V, Losos M, et al. The Newcastle-Ottawa Scale (NOS) for assessing the quality of nonrandomised studies in meta-analyses. Ottawa, ON: Ottawa Health Research Institute, University of Ottawa; 2015. http:// www.ohri.ca/programs/clinical_epidemiology/oxford.asp

33 Duval S, Tweedie R. Trim and fill: a simple funnel-plot-based method of testing and adjusting for publication bias in meta-analysis. Biometrics. 2000;56:455-63.

34 Hozo SP, Djulbegovic B, Hozo I. Estimating the mean and variance from the median, range, and the size of a sample. BMC Med Res Methodol. 2005;5:13.

35 Elwan MM, Abd Elghafar AE, Hagras SM, Abou Samra WA, Saleh SM. Long-term outcome of internal limiting membrane peeling with and without foveal sparing in myopic foveoschisis. Eur J Ophthalmol. 2019;29:6974.

36 Shiraki N, Wakabayashi T, Ikuno Y, Matsumura N, Sato S, Sakaguchi H, et al. Foveasparing versus standard internal limiting membrane peeling for myopic traction maculopathy: a Study of 102 Consecutive Cases. Ophthalmol Retina. 2020;4:1170-80. 20

37 Iwasaki M, Miyamoto H, Okushiba U, Imaizumi H. Fovea-sparing internal limiting membrane peeling versus complete internal limiting membrane peeling for myopic traction maculopathy. Jpn J Ophthalmol. 2020; 64:13-21.

38 Wang L, Wang Y, Li Y, Yan Z, Li Y, Lu L, et al. Comparison of effectiveness between complete internal limiting membrane peeling and internal limiting membrane peeling with preservation of the central fovea in combination with $25 \mathrm{G}$ vitrectomy for the treatment of high myopic foveoschisis. Medicine. 2019;98:e14710.

39 Gass JD. Müller cell cone, an overlooked part of the anatomy of the fovea centralis: hypotheses concerning its role in the pathogenesis of macular hole and foveomacualr retinoschisis. Arch Ophthalmol. 1999;117: 821-3. 
40 Hayashi K, Ohno-Matsui K, Shimada N, Moriyama M, Kojima A, Hayashi W, et al. Long-term pattern of progression of myopic maculopathy: a natural history study. Ophthalmology. 2010;117:1595-4.

41 Lee CL, Wu WC, Chen KJ, Chiu LY, Wu KY, Chang YC. Modified internal limiting membrane peeling technique (maculorrhexis) for myopic foveoschisis surgery. Acta Ophthalmol. 2017;95:e128-31.

42 Peng KL, Kung YH, Hsu CM, Chang SP, Tseng PL, Wu TT. Surgical outcomes of centripetal non-fovea-sparing internal limiting membrane peeling for myopic foveoschisis with and without foveal detachment: a follow-up of at least 3 years. Br J Ophthalmol. 2020;104:1266-70.
43 Meng B, Zhao L, Yin Y, Li H, Wang X, Yang $\mathrm{X}$, et al. Internal limiting membrane peeling and gas tamponade for myopic foveoschisis: a systematic review and meta-analysis. BMC Ophthalmol. 2017;17:166.

44 Yun LN, Xing YQ. Long-term outcome of highly myopic foveoschisis treated by vitrectomy with or without gas tamponade. Int J Ophthalmol. 2017;10:1392-5.

45 Chen X, Yao Y, Hao X, Liu X, Liu T. A comparative study of vitrectomy combined with internal limiting membrane peeling for the treatment of idiopathic macular hole with air or C3F8 intraocular tamponade. J Ophthalmol. 2018;2018:1672501.

46 Hejsek L, Stepanov A, Dusova J, Marak J, Nekolova J, Jiraskova N, et al. Microincision $25 \mathrm{G}$ pars plana vitrectomy with peeling of the inner limiting membrane and air tamponade in idiopathic macular hole. Eur J Ophthalmol. 2017;27:93-7.
47 Hasegawa Y, Hata Y, Mochizuki Y, Arita R, Kawahara S, Kita T, et al. Equivalent tamponade by room air as compared with $\mathrm{SF}(6)$ after macular hole surgery. Graefes Arch Clin Exp Ophthalmol. 2009;247:1455-9.

48 Usui $\mathrm{H}$, Yasukawa T, Hirano Y, Morita H, Yoshida M, Ogura Y. Comparative study of the effects of room air and sulfur hexafluoride gas tamponade on functional and morphological recovery after macular hole surgery: a retrospective study. Ophthalmic Res. 2013;50:227-30.

49 He F, Dong F, Yu W, Dai R. Recovery of photoreceptor layer on spectral-domain optical coherence tomography after vitreous surgery combined with air tamponade in chronic idiopathic macular hole. Ophthalmic Surg Lasers Imaging Retina. 2015;46: 44-8. 